\title{
Snow depth sensing using the GPS L2C signal with a dipole antenna
}

\author{
Qiang Chen*, Daehee Won and Dennis M Akos
}

\begin{abstract}
Prior research has shown that the currently deployed geodetic global positioning system (GPS) stations can be used to measure snow depth in an area around the antenna installation via GPS interferometric reflectometry (GPS-IR). Although such a technique provides the advantages of large spatial coverage and high temporal measurement sampling, there are also drawbacks in using geodetic equipment for this application. The geodetic antenna is costly and designed to mitigate the multipath signal, while for snow depth sensing, the reflective component contains the desired information regarding the environment. In this paper, the authors reviewed the principles of snow depth sensing via GPS-IR and show that the horizontally polarized electromagnetic wave is the desired signal for this application. A customized dipole antenna, which was designed to be horizontally polarized by properly adjusting its orientation, was built and evaluated in an experiment carried out at Table Mountain, Boulder, Colorado, during early February 2012. In this experiment, a universal software radio peripheral (USRP) is used to collect the raw data and a software-defined receiver (SDR) for L2C signal is used for data processing. Data and results from this dipole antenna/SDR implementation are compared with those from the geodetic GPS station and show an improvement for snow depth estimation.
\end{abstract}

Keywords: Snow depth; GPS-IR; L2C; Dipole antenna; Remote Sensing

\section{Introduction}

Seasonal snow cover is an important component in the investigation of the land surface climate and hydrology. The measurement of the amount of water stored in the snowpack and forecasting the rate of melt are essential for management of the water supply and flood control systems [1]. It has been shown that the current network of geodetic global positioning system (GPS) stations, which were installed for geophysical and surveying applications, are capable of measuring the snow depth in their vicinity using GPS interferometric reflectometry (GPS-IR) providing a large spatial footprint with a high update rate [2]. The principle for GPS-IR leverages the relationship between the variations of the GPS signal power, i.e., the signal-to-noise ratio (SNR) and the snow depth. Currently, there are 32 GPS satellites in operation and the period of a GPS satellite is approximately $24 \mathrm{~h}$, which results in 64 available tracks (ascending and descending track) per day. The snow depth estimation via GPS-IR would process a

\footnotetext{
* Correspondence: qiang.chen@colorado.edu

Department of Aerospace Engineering and Sciences, University of Colorado, Boulder, CO 80309-0553, USA
}

period of SNR data within a particular elevation angle range and obtain a snow depth estimate for each track. Figure 1 from [2] shows the SNR before and after a $35-\mathrm{cm}$ snowfall at Marshal, Colorado, from a geodetic GPS station. The signal coherently reflected off the ground coupled with the direct signal results in variations of the received GPS signal power. The height between the GPS antenna and the reflection surface determines the specific frequency, which can be used to infer the height of the antenna and, hence, the snow depth. The data from the EarthScope Plate Boundary Observatory ( $\mathrm{PBO}$ ), which was built to monitor Earth deformation and has more than 1,100 geodetic GPS stations across North America, has been leveraged to provide snow depth measurements which are publicly available [3].

However, there are two drawbacks in using geodetic GPS equipment to measure snow depth. First, existing geodetic GPS stations are expensive and thus expanding coverage to new sites, specifically for snow depth monitoring, is likely not the best fiscal approach. Second, and more importantly, the geodetic antenna is not optimal for the snow depth sensing application. Multipath is a

\section{Springer}




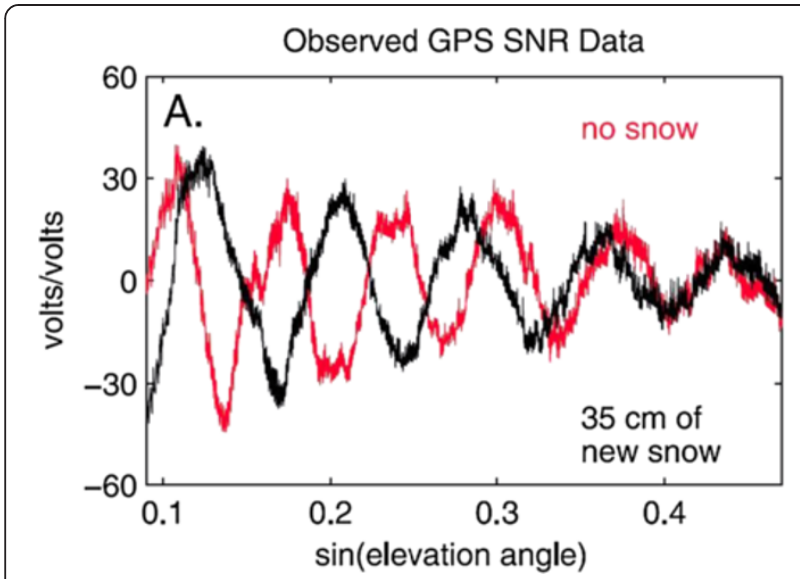

Figure 1 Geodetic SNR before and after a 35-cm snowfall [2].

dominant error source for GPS positioning, and the current geodetic GPS antennas have been designed to minimize the reception of the reflected signal by suppressing both the left hand circular polarized (LHCP) and low elevation angle components for high-precision positioning. Since the broadcast GPS signal is right hand circular polarized (RHCP) [4], the reflected signal will be depolarized resulting in an LHCP component. The distribution of the RHCP and LHCP components will be based on the properties of reflection surface and the grazing angle of the incident wave. Additionally, the geodetic antenna has a low gain pattern for low elevation angle incident signals, which is a further design element to suppress the groundreflected signal. In contrast, snow depth sensing utilizes the ground-reflected component to make the signal power variations more distinct. This limitation of the geodetic antenna for snow depth sensing is illustrated in [5] and thus makes it far from the optimal design in this application. This is not to say that the EarthScope PBO snow monitoring is not effective as it works quite well far extending the capabilities of that network, rather, if the GPS signal is to be used primarily as a snow sensor, an alternate hardware configuration can likely provide higher performance with lower costs.

In [6], a new civil signal on the L2 frequency designated L2C has shown better performance than the traditional L1 C/A signal for snow depth sensing. The reason for this superiority is that the increased code length of L2C decreases the auto-correlation and cross-correlation sidelobes relative to the $\mathrm{C} / \mathrm{A}$ signal. This property is desirable because it decreases the influence of the multipath signal's sidelobes in the SNR.

Previous research has proved the viability and superiority of measuring snow depth via GPS-IR by comparing the measured snow depth with those from manual measurement, ultrasonic, SNOTEL, LIDAR, and laser sensors $[2,6,7]$. The factors that impact the observables from geodetic GPS stations are analyzed in [4]. Although it is recognized that geodetic GPS stations are not optimal for snow depth sensing via GPS-IR, current research has not illustrated what are the required characteristics for an optimal antenna to be used in snow depth sensing via GPS-IR. In this paper, we analyzed the desired characteristics for the incident electromagnetic wave and presented a custom dipole antenna which satisfied many of the criteria for this application. Also, we implemented a software-defined receiver (SDR) for L2C which can provide maximum flexibility. An experiment was carried out to evaluate the performance of the custom dipole antenna by comparing the results with those from a geodetic GPS station.

This paper is organized as follows. Section 2 reviews the principle of snow depth sensing via GPS-IR. In Section 3 , the desired features for the incident electromagnetic wave are presented by analyzing the reflection coefficients of different polarizations. In Sections 4 and 5, details are presented on the setup and implementation of the data collection and processing tools, especially the custom dipole antenna. In Section 6, the experiment results are presented and compared with those from a geodetic GPS station. Section 7 concludes that the dipole antenna shows the potential for more accurate snow depth estimate compared to geodetic GPS stations.

\section{SNR and multipath tool}

Since the SNR is our primary observable in the snow depth sensing application, it is necessary to introduce the concept of SNR and the influence of multipath signal. In the GPS receiver, SNR is used as an indicator of the signal power. Typically, the SNR estimate is derived from the output of the carrier tracking loop. In the carrier tracking loop, both an in-phase and a quadrature carrier replica, which have a $90^{\circ}$ phase difference, are generated to correlate with the received signal. Accordingly, an in-phase correlation (I) and a quadrature correlation (Q) are obtained. The carrier tracking loop attempts to allocate all the signal energy to the I channel and only noise to the Q channel by adjusting the carrier frequency of the local replica. Figure 2 shows the effect of multipath on the carrier phase measurement in the absence of noise [8].

If we assume that the antenna works on a particular dominant polarization (only receive the incident wave with a particular polarization), the composite SNR can be described by [9]:

$$
\mathrm{SNR} \equiv A_{d}+A_{r}+2 \sqrt{A_{d} A_{r}} \cos \phi
$$

where $A_{d}$ and $A_{r}$ are the power of the direct signal and reflected signal, respectively, $\phi$ is the angle between the direct phasor and the reflected phasor. 


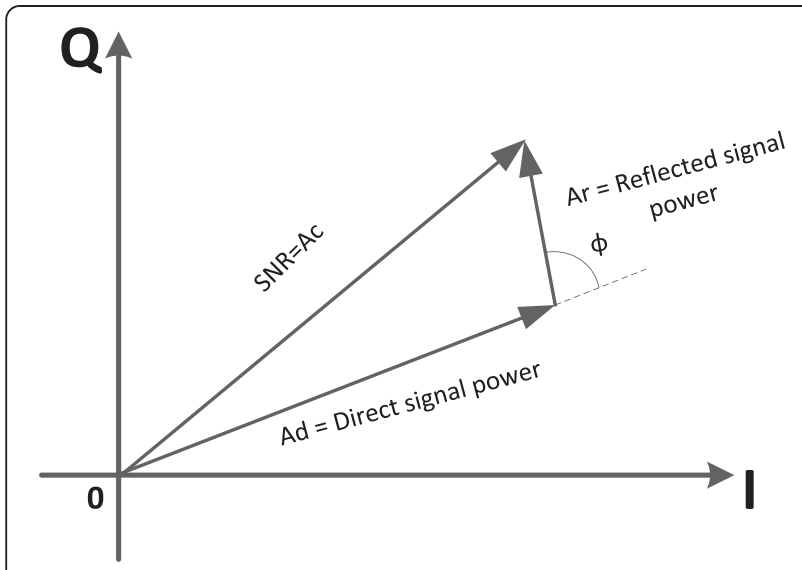

Figure 2 Effect of multipath on carrier phase in a GPS receiver [8].

The change of $\phi$ determines the oscillation frequency of the received SNR. In [10-13], the relationship between carrier phase error and SNR for short-delay multipath has been illustrated in detail. Here, we reiterate the most important equations. The total interference angle $\phi$ consists of three components, which can be given by

$$
\phi=\phi_{g}+\phi_{r}+\phi_{a}
$$

where $\phi_{g}, \phi_{r}$ and $\phi_{a}$ correspond to: the interference angle introduced by the geometry of the antenna/reflector (shown in Figure 3), the reflection of the incident wave, and the possible different antenna phase pattern for incident wave from different elevation angles, respectively. From Figure $3, \phi_{g}$ can be computed by

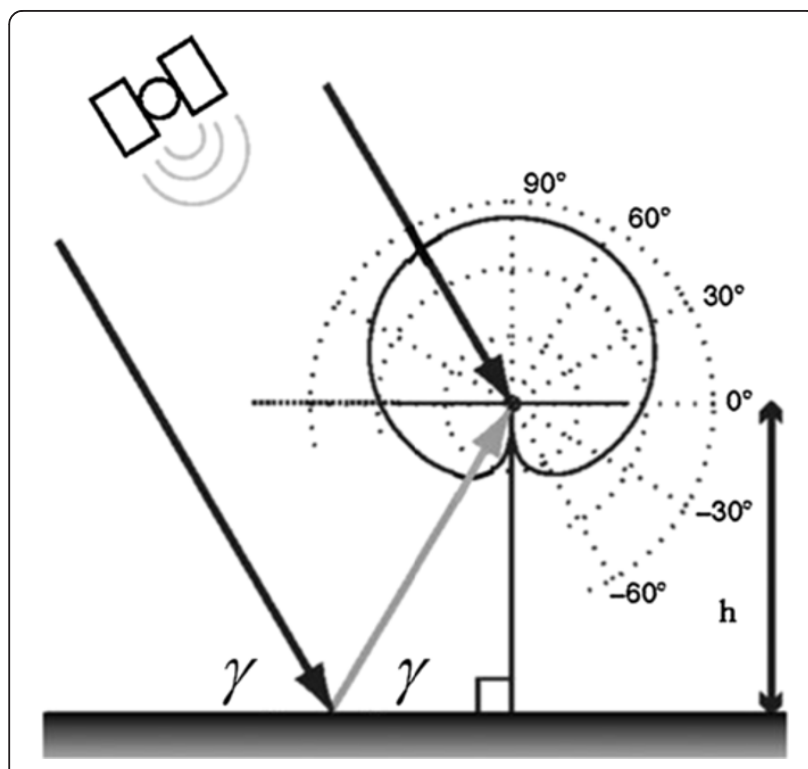

Figure 3 Geometric relationship of the direct signal and reflected signal [8]. A typical geodetic antenna pattern for RHCP is sketched.

$$
\phi_{g}=\frac{4 \pi h \sin \gamma}{\lambda}
$$

where $\gamma$ is the grazing angle (equal to the elevation angle if the reflection surface is horizontal), $h$ is the perpendicular distance from the antenna to the reflection surface, $\lambda$ is the wavelength of the frequency (L2 is $1,227.6 \mathrm{MHz}$, and $\lambda_{L 2}$ is $24.4 \mathrm{~cm}$ ). Please note that $\phi_{g}$ is related to the antenna height $h$ which is our interest; thus, $h$ can be retrieved from the observed SNR.

$\phi_{r}$ is the extra interference angle introduced by the reflection off the ground, which equals the phase of the reflection coefficient with respect to the particular polarization of the incident wave. $\phi_{r}$ depends on the grazing angle and the polarization of the incident wave as well as the characteristics of the ground medium. $\phi_{a}$ is the interference angle introduced by the possible different phase patterns for the direct incident wave with a positive elevation angle and the reflected wave with a negative elevation angle. If the phase pattern of the antenna is constant with respect to the elevation angle which is in the range to be used in the snow depth sensing application, then $\phi_{a}$ is zero.

To eliminate the time-varying effect, the definition:

$$
x=\sin \gamma, f_{M}=2 h / \lambda
$$

is used to obtain:

$$
\phi=2 \pi f_{M^{x}}+\phi_{r}+\phi_{a}
$$

where $f_{M}$ is defined as the spatial frequency [12]. If $f_{M}$ can be estimated from the observed SNR, then the simple planar reflector distance can be computed as:

$$
h=\frac{1}{2} f_{M} \lambda
$$

If the antenna heights $h_{1}$ and $h_{2}$ which correspond, respectively, to the cases before and after a snowfall can be obtained, then the snow depth can be computed by $\Delta h=h_{1}-h_{2}$. From Figure 1, an obvious frequency/height change in the SNR resulting from the $35-\mathrm{cm}$ snowfall can be observed.

From (1) and (5), we can see that if the interference angle $\phi_{r}$ and $\phi_{a}$, as well as the signal power $A_{d}$ and $A_{n}$ are constant within the observed elevation angle range, then, the SNR will be purely sinusoidal and the spatial frequency $f_{M}$ can be estimated using frequency domain transfer algorithm. Otherwise, changes of $\phi_{n} \phi_{a}, A_{d}$, and $A_{r}$ will blur the resulting frequency of the SNR and increase the difficulty to estimate $f_{M}$. If $A_{d}$ and $A_{r}$ change in a slow non-oscillatory way (as shown in Figure 1) with the elevation angle, it would not affect the estimate of $f_{M}$. However, any change in $\phi_{r}$ and $\phi_{a}$ will affect the estimation of $f_{M}$ and this will be illustrated in detail in Section 3. 


\section{The desired characteristics for the optimal antenna} From previous discussion, the optimal antenna should have the following two characteristics. First, it has the ability to keep $\phi_{r}$ and $\phi_{a}$ constant across the observed elevation angle range. Second, it would balance the received direct and reflected signal powers and keep these constant across the observed elevation angle range.

To analyze the interference angle $\phi_{r}$ it is necessary to analyze the reflection coefficients with respect to different polarizations of the incident electromagnetic wave. Although the broadcast GPS signals are RHCP, it is reasonable to decompose the RHCP wave into the combination of vertically polarized and horizontally polarized waves. These two kinds of linearly polarizations are illustrated in Figure 4.

The reflection coefficients for vertical and horizontal polarization (assume specular reflection) are given by [14].

$$
\begin{aligned}
R_{v} & =\frac{\eta_{1} \cos \theta_{t}-\eta_{0} \cos \theta_{i}}{\eta_{0} \cos \theta_{i}+\eta_{1} \cos \theta_{t}} \\
R_{h} & =\frac{\eta_{1} \cos \theta_{i}-\eta_{0} \cos \theta_{t}}{\eta_{1} \cos \theta_{i}+\eta_{0} \cos \theta_{t}}
\end{aligned}
$$

where $\theta_{i}$ is the angle of incidence and $\theta_{t}$ is the angle of refraction. Both $\theta_{i}$ and $\theta_{t}$ are relative to the normal direction, and $\theta_{i}$ is the complement of the grazing angle $\gamma$ under the assumption that the reflection surface is horizontal. $\eta_{0}$ and $\eta_{1}$ are the intrinsic impedances of free-space (air) and the ground (soil or snow), respectively. The definitions of $\eta_{0}$ and $\eta_{1}$ are as follows:

$$
\begin{aligned}
& \eta_{0}=\sqrt{\frac{\mu_{0}}{\varepsilon_{0}}} \\
& \eta_{1}=\sqrt{\frac{j \omega \mu_{1}}{\sigma_{1}+j \omega \varepsilon_{1}}}
\end{aligned}
$$

The angle of incidence $\theta_{i}$ and the angle of refraction $\theta_{t}$ can be related by Snell's law

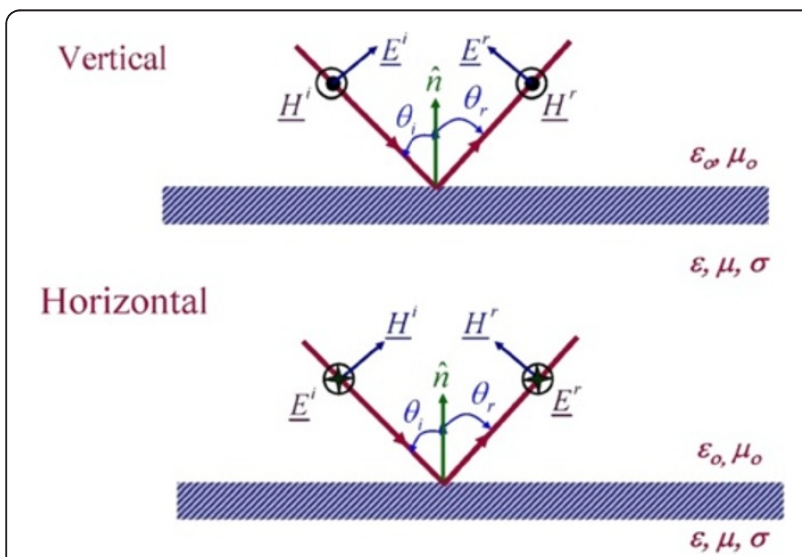

Figure 4 Vertical polarization and horizontal polarization.

$$
\begin{aligned}
& \gamma_{0} \sin \theta_{i}=\gamma_{1} \sin \theta_{t} \\
& \gamma_{0}=j \omega \sqrt{\varepsilon_{0} \mu_{0}} \\
& \gamma_{1}=\sqrt{j \omega \mu_{1}\left(\sigma_{1}+j \omega \varepsilon_{1}\right)}
\end{aligned}
$$

where $\varepsilon_{0}$ and $\mu_{0}$ are the permittivity and permeability, respectively, of the upper medium; $\varepsilon_{1}, \mu_{1}$, and $\sigma_{1}$ are the permittivity, permeability, and conductivity, respectively, of the bottom medium (the bottom medium occupies the entire lower hemisphere); $\omega$ is the angular frequency; $j$ is the imaginary unit. Generally, the complex relative permittivity $\varepsilon_{r}$ which is defined as

$$
\varepsilon_{r}=\varepsilon_{1} / \varepsilon_{0}=\varepsilon_{r}^{\prime}+j \varepsilon_{r}^{\prime \prime}=\varepsilon_{r}^{\prime}-j 60 \lambda \sigma
$$

is more often used in the remote sensing literature.

Here, we simulated the reflection coefficients for both the vertical and the horizontal polarizations at different grazing angles for dry soil $\left(\varepsilon_{r}^{\prime}=4.2, \sigma=0.0005\right)$, wet soil $\left(\varepsilon_{r}^{\prime}=10, \sigma=0.0008\right)$, and snow $\left(\varepsilon_{r}^{\prime}=2.2, \sigma=0.00005\right)$, which are shown in Figures 5 and 6 , respectively.

For the vertical polarization, the amplitude of the reflection coefficient decreases to zero rapidly at a particular angle, which is known as the Brewster's Angle. Recognize that the phase of the reflection coefficient (i.e., $\phi_{r}$ ) also experiences a sharp change from $0^{\circ}$ to $180^{\circ}$ around the Brewster's Angle. As stated previously, the change of $\phi_{r}$ would distort the spatial frequency $f_{M}$ (i.e., the snow depth) and hence should be avoided, especially due to the fact that the Brewster's Angle can fall into the elevation angle range (usually low elevation angles are used to provide a large footprint) with a large probability. So, the vertically polarized wave is not suitable for snow depth sensing via GPS-IR. In contrast, the phase of the reflection coefficient
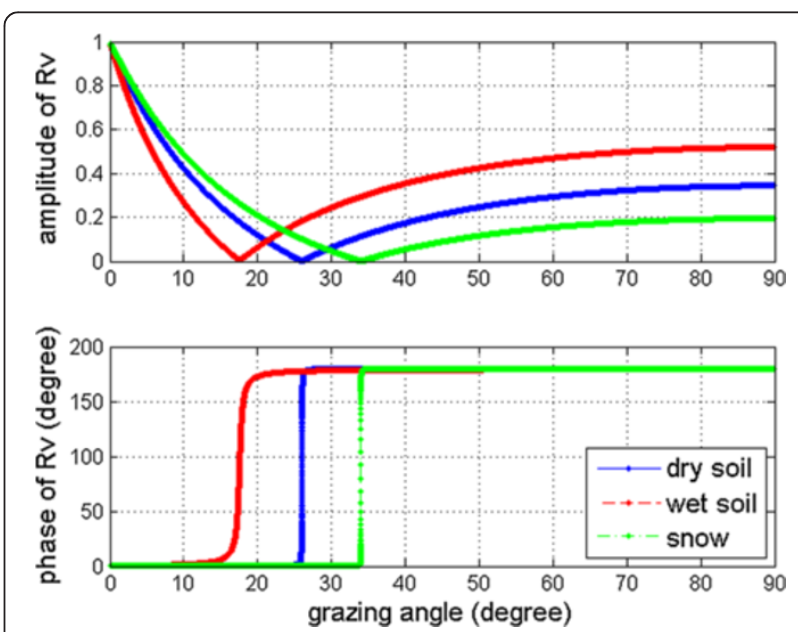

Figure 5 Reflection coefficient (amplitude and phase) for vertical polarization. 

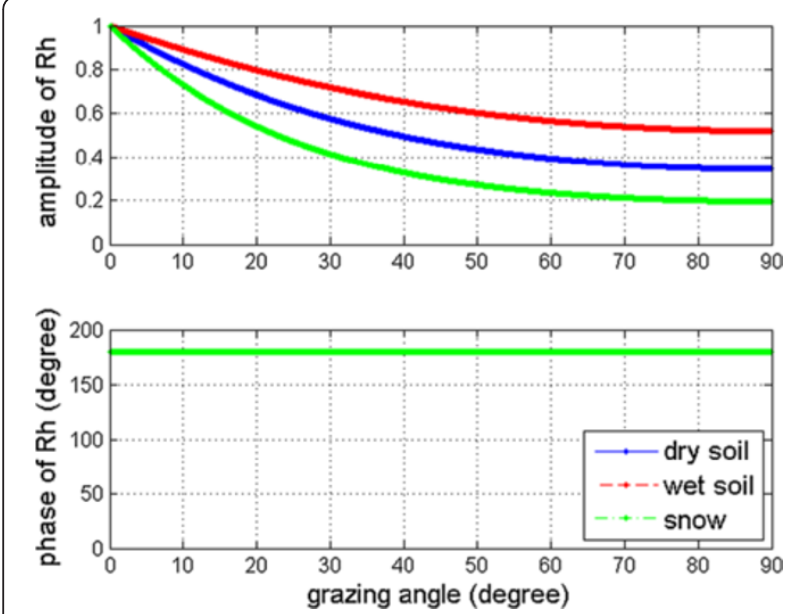

Figure 6 Reflection coefficient (amplitude and phase) for horizontal polarization.

for the horizontal polarization is constant over the full elevation angle range. Also, the reflected power is relatively large compared to the vertical polarization, especially at low grazing angles. From the above analysis, the desired antenna for snow sensing via GPS-IR should be horizontally polarized.

Although the power density of GPS signal changes slightly (within $2.5 \mathrm{~dB}$ ) with respect to the elevation angle [15], it is a reasonable approximation that the direct signal power density is constant. The requirement for the optimal antenna to keep the direct and reflected power constant can be explained as keeping the radiation pattern constant in the upper hemisphere and compensating the loss of reflection power with respect to elevation angles in the bottom hemisphere. This is too strict of a requirement and hence is very difficult to implement in antenna design and fabrication. Considering the fact that a slow and non-oscillatory change in amplitude does not affect the retrieval of the spatial frequency $f_{M}$, it is prudent to focus the requirements for the antenna on the horizontal polarization selection.

\section{Custom implementation}

\subsection{Dipole antenna}

The horizontal polarization is the key requirement for the antenna design. Among the typical antenna types, the loop antenna is horizontally polarized for all the azimuths and thus serves as a favorable option. However, the internal loop/wire inductance makes the loop antenna difficult to be matched [14]. To simplify the antenna fabrication, a customized wire antenna was designed and built, which is given in Figure 7. The simulated radiation pattern of this antenna, which is shown in Figure 8, approximates a theoretical half wavelength dipole antenna and it can be matched easily by adjusting the bending angles of the four wire legs [16]. For

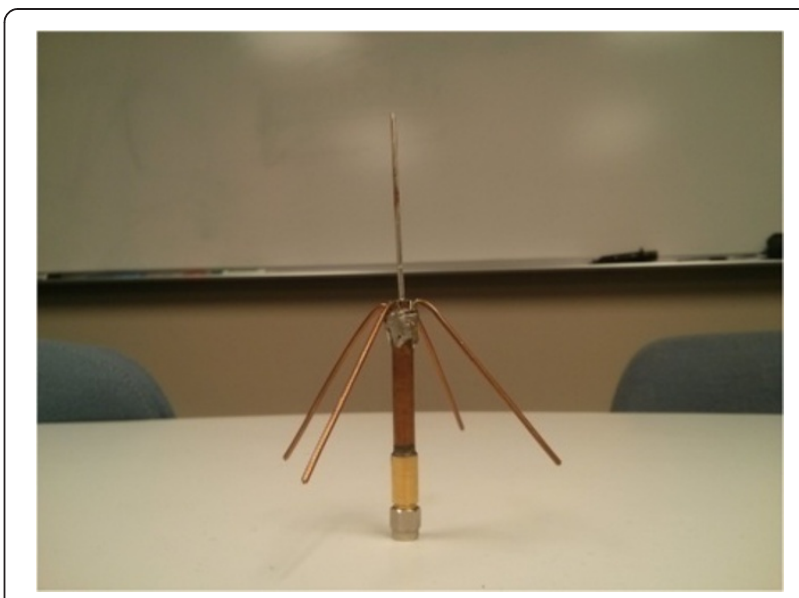

Figure 7 Home-built L2 dipole antenna.

simplicity, it will be referred as dipole antenna in the later paragraphs. The half wavelength dipole antenna is well modeled in antenna theory; thus, the important characteristics related to the snow sensing are listed below.

\subsubsection{Radiation pattern}

The radiation pattern of the half wavelength dipole antenna is as follows:

$$
U(\theta, \phi) \approx A_{0} \sin ^{3} \theta
$$

where $\theta$ is the zenith angle and $\phi$ is the azimuth angle in spherical coordinate frame. If the radiation pattern is irrelevant to $\phi$, this antenna is referred as an omnidirectional antenna. Please note that there are two nulls at $\theta=0^{\circ}$ and $\theta=180^{\circ}$ and also the maximum radiation intensity locates at $\theta=90^{\circ}$.

\subsubsection{Vector effective length}

The vector effective length of the half wavelength dipole antenna is given by

$$
\vec{l}_{\text {eff }}=-\hat{a}_{\theta} \frac{\lambda}{\pi} \frac{\cos \left(\frac{\pi}{2} \cos \theta\right)}{\sin \theta}
$$

Where $\hat{a}_{\theta}$ is the unit vector in the direction of zenith angle in spherical coordinate frame. For a receiving antenna, the open circuit voltage $V_{\text {oc }}$ stimulated by the incident electric field $\vec{E}_{\text {inc }}$ can be calculated by

$$
V_{\text {oc }}=\vec{E}_{\text {inc }} \cdot \vec{l}_{\text {eff }}
$$

Note that both $\vec{E}_{\text {inc }}$ and $\vec{l}_{\text {eff }}$ are vectors and only if they are in the same direction will $V_{\mathrm{oc}}$ reach its maximum value. The polarization efficiency $p_{e}$ which is given by

$$
p_{e}=\frac{\left|\vec{l}_{\mathrm{eff}} \cdot \vec{E}_{\mathrm{inc}}\right|}{\left|\vec{l}_{\mathrm{eff}}\right|^{2}\left|\vec{E}_{\mathrm{inc}}\right|^{2}}
$$




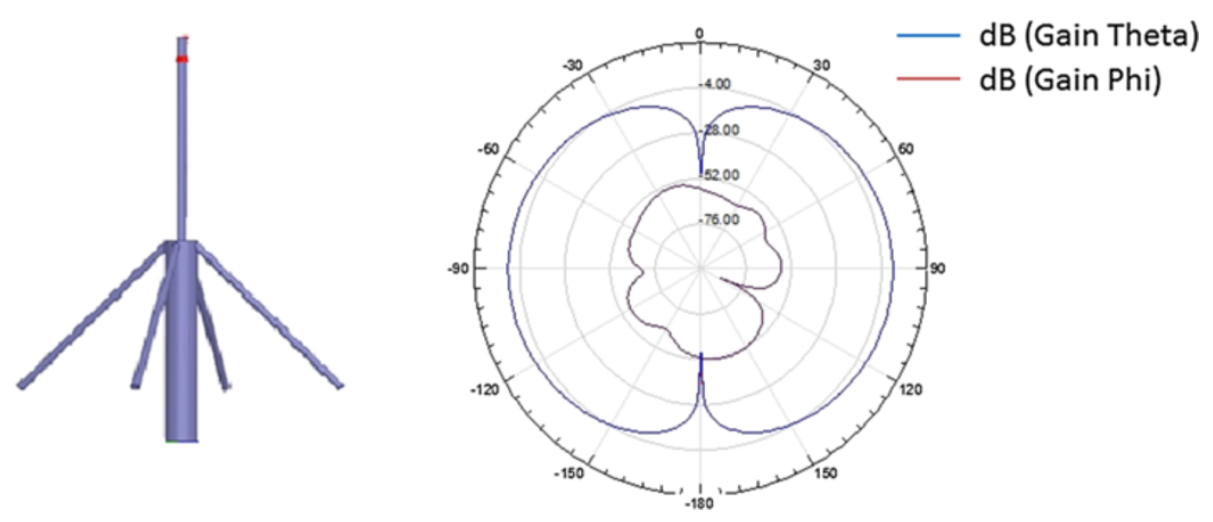

Figure 8 ANSYS HFSS model and simulated radiation pattern.

is used to describe this polarization mismatch between the incident electric field and the receiving antenna.

From (15), this dipole antenna will be vertically polarized if it is placed upright. The simulated gain pattern in Figure 8 verifies the validity of the half wavelength dipole model. The simulation shows that the gain in the phi direction (corresponding to the horizontal polarization gain if the antenna is placed upright) is negligible compared to the gain in theta direction (corresponding to the vertical polarization gain if the antenna is placed upright). If this dipole antenna is placed upright, the vertical component of the L2C signal will be received exclusively and the horizontal component will be rejected, which is against to our intension.

However, if this antenna is tipped, it will be horizontally polarized at the direction $\theta=90^{\circ}$ (here, $\theta$ is the zenith angle of the antenna), which is the desired polarization for the desired antenna. Please note that since the radiation pattern is omnidirectional, the phase response for the direct and reflected horizontally polarized waves are also constant, and thus $\phi_{a}$ is zero.

This dipole antenna is made up of a hardline coax cable, stripped of its outside copper layer to expose the inner pin and soldered four copper wire legs on it. The length of the inner pin and the legs is $5.5 \mathrm{~cm}$ and the bending angle is $45^{\circ}$. A network analyzer was used to tune the design, locating the null of $\left|S_{11}\right|$ ( $S_{11}$ is the complex reflection coefficient of one-port network) at the L2 frequency by adjusting the length of pin and the bending angles of the four legs.

\subsection{Software-defined receiver}

\subsubsection{Front end - universal software radio peripheral}

A universal software radio peripheral (USRP) N210 platform from Ettus Research Inc. (Santa Clara, CA, USA) is shown in Figure 9. This device served as the raw intermediate frequency (IF) data collection platform from which the software defined GPS L2C receiver was developed.

The USRP has the following features that make it a powerful RF/IF data collection tool.
- Wide frequency range from 0.8 to $2.35 \mathrm{GHz}$, which covers all the GPS frequencies (L1, 1,575.42 MHz; L2, 1,227.6 MHz; L5, 1,176.45 MHz).

- Sampling rates is up to $50 \mathrm{Msps}$ and wide RF bandwidth $(25 \mathrm{MHz})$, which enables low-loss sampling for any GPS signal.

- 14-bit ADCs/DACs enable high dynamic range and quantization resolution.

- The USRP has a $1 \mathrm{~Gb}$ Ethernet interface which enables data transfer at any sampling rate and bit level.

As the L2C signal is of interest, the center frequency and sampling rate are set to $1,227.5 \mathrm{MHz}$ (giving an IF of $100 \mathrm{KHz}$ ) and $2 \mathrm{Msps}$, respectively.

\subsubsection{Signal processing}

A SDR has been developed to process the raw IF data collected by the USRP to provide maximum flexibility into the receiver processing. It is an extension of the open source L1 Matlab receiver documented in [17]. L2C is the new civil code signal transmitted at L2 frequency $(1,227.6 \mathrm{MHz})$ with the same chipping rate as the L1 $\mathrm{C} / \mathrm{A}$ code. This new civil signal is available on only a subset of the current GPS constellation (on the current/future Block IIR-M, Block IIF and Block III satellites). At the time of the experiment, there were nine GPS satellites (PRN 1, 5, 7, 12, 15, 17, 25, 29, and 31) that broadcast this

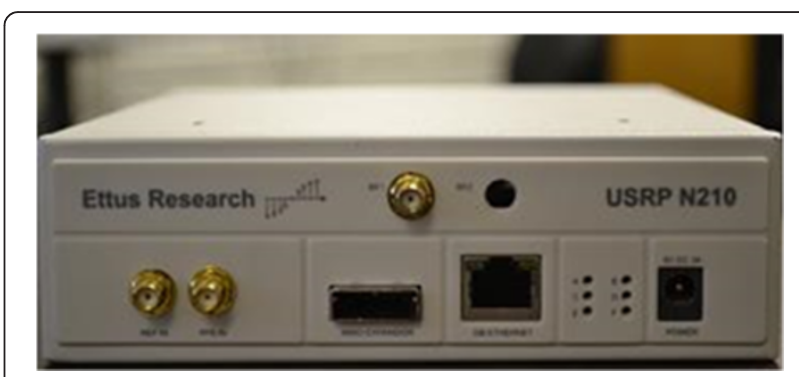

Figure 9 USRP N210. 
new signal. The L2C signal has the following advantages for snow sensing compared to L1 C/A code, which is demonstrated in [6].

- L2C has superior code cross correlation (40 dB compared to CA's $24 \mathrm{~dB}$ ) and continuous wave interference rejection.

- $\mathrm{L} 2 \mathrm{C}$ has a pilot (data-free) channel for extended integration time.

- Improved data structure for enhanced data demodulation offers a 5-dB improvement compared to the $\mathrm{L} 1 \mathrm{C} / \mathrm{A}$.

In a typical SDR, the delay lock loop (DLL) and phase lock loop (PLL) are used to track the code phase and the carrier phase of the received signal. However, the dipole antenna has no suppression of the reflected signal so the combined SNR could be very low when the direct signal and the reflected signal are of opposite phases. The traditional tracking loop may lose lock when the reflected signal degrades the direct signal. An open loop (OL) tracking is an alternative to the traditional tracking loop that can provide additional sensitivity. The OL tracking does not use feedback loops but rather performs repetitive acquisitions at a particular time interval. The OL tracking provides a more robust tracking approach than the traditional closed loop tracking and avoids the need for tracking thresholds and reacquisition algorithms. An advantage of this USRP/SDR implementation is that SNR estimates are available at a much higher update rate than the geodetic GPS receivers (which typically operate at $1 \mathrm{~Hz}$ ) and the high-rate SNR can be averaged to obtain lower noise measurement.

\section{Experimental setup}

An ongoing field experiment was running during 2 to 4 Feb. 2012 to assess the potential of this design for measuring the height of antenna above the ground on the Table Mountain plateau north of Boulder, Colorado $\left(105^{\circ} 13.96^{\prime} \mathrm{W}, 40^{\circ} 7.81^{\prime} \mathrm{N}\right)$. Conveniently, it was operating during an early February snowfall and this provided the opportunity to assess its potential as a snow depth measurement system. A block diagram of the experimental setup is shown in Figure 10, and photos are shown in Figure 11. The dipole antenna was east oriented, so the SNR of the satellite whose ground track ran south/north oriented was the focus.

There were two primary data sets collected in parallel for this experiment. First are the raw IF samples from the USRP connected to the L2 dipole antenna (with amplifier and filter). The L2C SDR code is used to process these collected samples. For comparison, a second data collection chain consisting of a Trimble geodetic antenna (Ashtech D/M Choke) and a Trimble

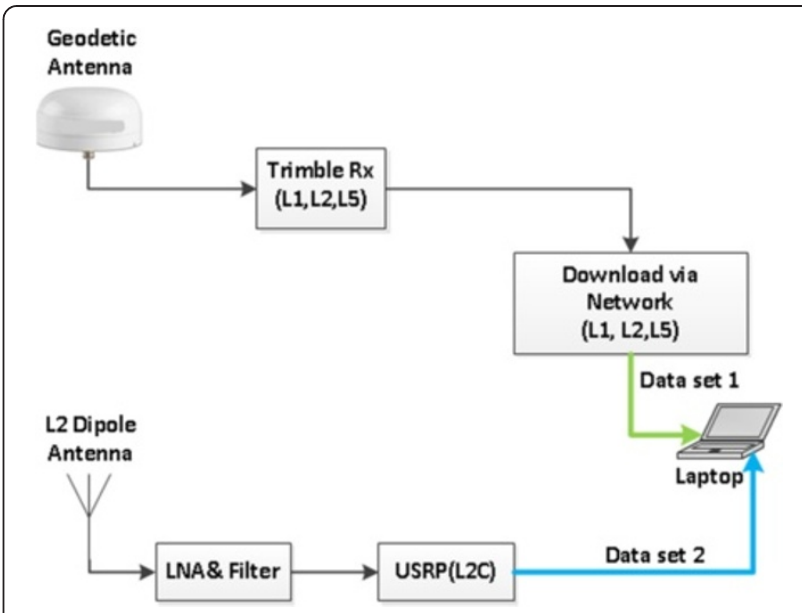

Figure 10 Block diagram of the data recording system.

NetR5 receiver were utilized to collect a parallel data stream. The two antennas were not collocated but in relative close proximity with the geodetic antenna approximately $15 \mathrm{~m}$ to the $\mathrm{SW}$ as shown in Figure 12 .

Again, the purpose of this experiment was to evaluate the performance of the customized dipole antenna in snow depth sensing via GPS-IR. The reason to use the USRP and SDR is that there was no readily available inexpensive L2C receiver with the desired flexibility. Although the USRP and the data storage devices (Lenovo Thinkpad x220 laptop and a 1 TB hard drive) were not inexpensive, the SDR was able to provide SNR measurements at a higher update rate and provided full control of the receiver processing. It is expected that low cost mass market receiver architectures will be well suited for this application once they become available for the GPS L2C signal.

\section{Results}

\subsection{SNR results}

As stated earlier, SNR is the observable for snow depth sensing via GPS-IR. Here, the SNR of PRN 1, 5, 17, 25 from the geodetic receiver are shown in Figure 13. They are offset within the figure so that they can be seen clearly. They are all ascending tracks, and the specular ground traces for all the four PRNs are shown in Figure 12. The ground tracks extend from 3 to $23 \mathrm{~m}$ in radius and the height of the geodetic antenna is approximately $2 \mathrm{~m}$. From Figure 14, we can see that the oscillations of the SNR are more distinct when the elevation angle is low. In contrast, the oscillations diminish when the elevation angles rise above $25^{\circ}$, implying that the reflective component is significantly attenuated at these higher angles. The reflective wave will contain more LHCP component as the elevation angle increases and the gain for the LHCP wave is usually much smaller than the gain for the RHCP wave for a geodetic antenna. Also, the RHCP gain for a reflective wave incoming from a negative elevation angle is much smaller 


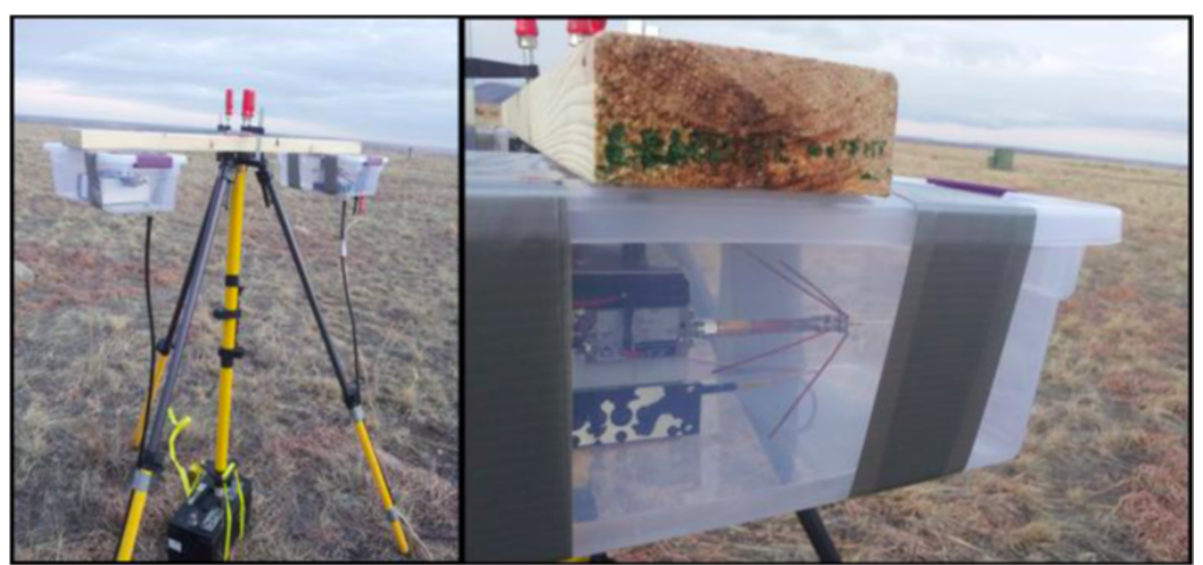

Figure 11 Data recording instrument on Table Mountain, Boulder, CO.

than the gain for an incoming direct wave from a positive elevation angle. The reasons for the behavior of geodetic SNR have been explained in detail in [4].

The SNR from PRN 05 is used for the dipole antenna as it has the best oriented alignment. Unfortunately, as shown in Figure 13, this PRN has limited oscillations in the SNR from the geodetic receiver and as a result PRN 17 is used for comparison to show the best possible for this implementation. The oscillations of the dipole SNR are more distinct (especially at high elevation angles) and more pure sinusoidal behaved, which is desirable for the spatial frequency (i.e., reflector height) retrieval.

The Lomb-Scargle periodograms (LSP) [18] of the two data sets, utilizing elevation angles from $5^{\circ}$ to $30^{\circ}$, are shown in Figure 15 . Above $30^{\circ}$, the oscillation pattern of the geodetic SNR diminishes and further hinders its estimation capabilities. A two-order polynomial model is used to fit and remove the rising trend of the geodetic SNR data. However, because the oscillations of the SNR

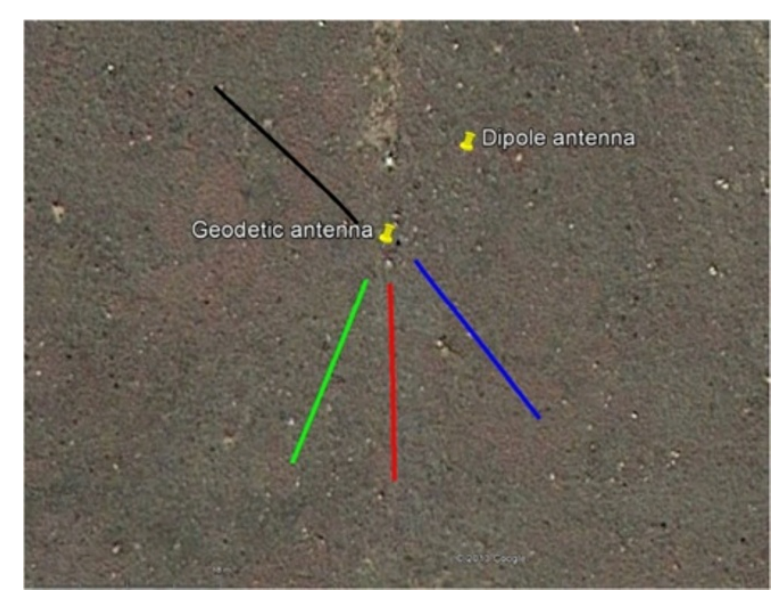

Figure 12 Ground traces of PRN 1, 5, 17, 25 (same color coding as in Figure 13). from the dipole antenna are still distinct at high elevation angles, it is possible to use the SNR data at a wider range of elevation angles to estimate the snow depth which will provide a longer reflection track. It can be clearly seen that the frequency peak is sharper for the SNR from the dipole antenna/SDR than that from the geodetic antenna/receiver. The reason for the different heights is the baseline heights/ locations of each antenna. The computed geodetic antenna height is $2.00 \mathrm{~m}$, and the computed dipole antenna height is $1.36 \mathrm{~m}$. The height resolution of the LSP is $3 \mathrm{~mm}$, which is smaller than the roughness of the ground.

Once the spatial frequency $f_{M}$ is obtained, the least square algorithm is used to fit the observed SNR using $f_{M}$. As stated previously, the observed SNR will be sinusoidal under the assumption that both the direct and reflected power are constant, as well as the phase of reflection coefficient remains constant. Considering this, it would be prudent to use the RMS of the fit residuals as

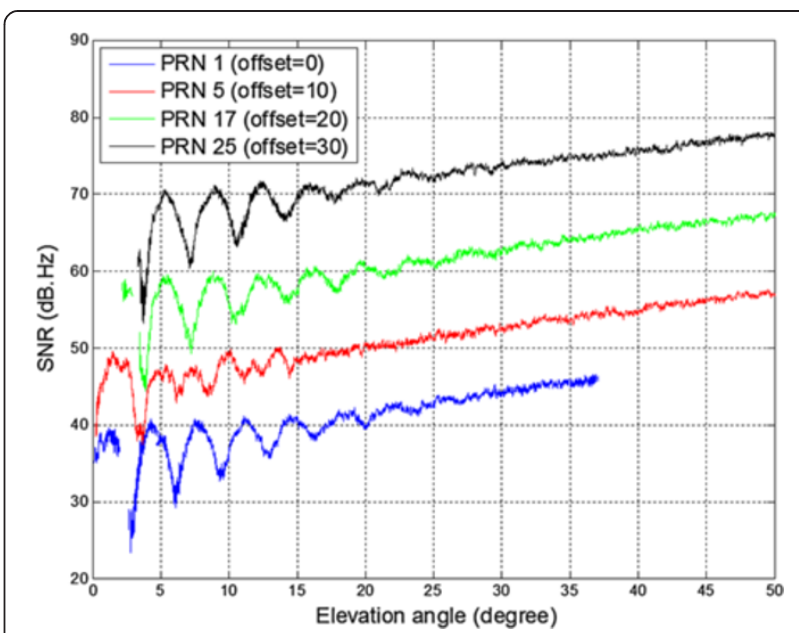

Figure 13 SNR of PRN 1, 17, 25 vs. elevation angle, offset for clarity. 


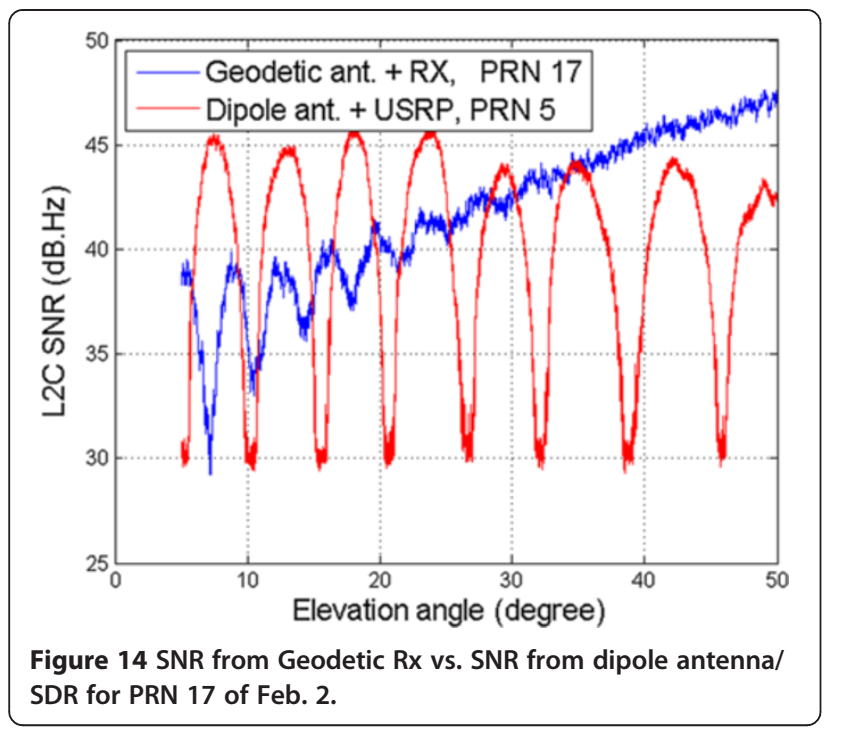

an indicator the goodness of fit, i.e., the confidence level of our estimate. To exclude the effect of the different amplitude levels of fit sinusoid, the normalized RMS of the fitted residuals

$$
\mathrm{RMS}_{\text {normalized }}=\frac{\mathrm{RMS}_{\text {fit }}}{A_{\text {fit }}}
$$

is used instead of its absolute value, where $\mathrm{RMS}_{\text {fit }}$ is the RMS of the fit residuals and $A_{\mathrm{fit}}$ is the amplitude of the fitted sinusoidal. The normalized RMS of the fit residuals are 0.45 and 0.23 for the geodetic data and the dipole antenna/SDR data, respectively, which clearly indicates that the dipole data will result in a better estimate with a higher confidence level.

Another metric which can be used to assess the accuracy is the $95 \%$ confidence interval (CI) of the estimated

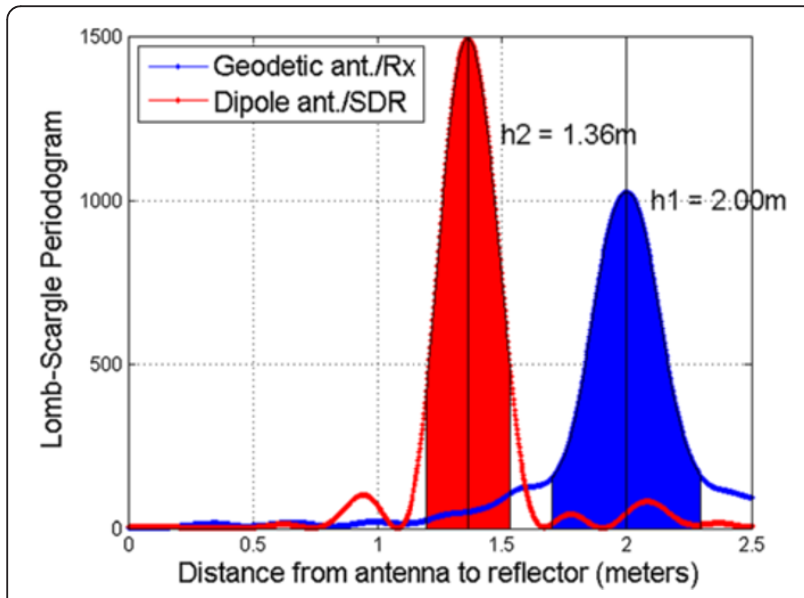

Figure 15 Antenna heights from geodetic SNR and dipole antenna/SDR.
Table 1 Heights estimation and corresponding 95\% Cls for L2C PRNs

\begin{tabular}{lll}
\hline PRN & Height $(\mathbf{m})$ & $\mathbf{9 5 \%} \mathbf{C l}(\mathbf{m m})$ \\
\hline 1 & 2.08 & 9.7 \\
7 & 2.00 & 9.6 \\
12 & 2.08 & 18.0 \\
15 & 2.40 & 16.7 \\
25 & 2.03 & 18.1 \\
29 & 2.03 & 17.6 \\
31 & 2.17 & 9.9 \\
\hline
\end{tabular}

height. Here, the bootstrapping resampling method is used to calculate the $95 \%$ CI of the height [19]. The 95\% CIs of the height estimation for the dipole and geodetic antenna data are 4.9 and $9.8 \mathrm{~mm}$, respectively. To better understand the performance of the geodetic data, the height estimates and corresponding 95\% CIs for different PRNs are listed in Table 1. We can see that the 95\% CIs for the geodetic data are approximate twice of $95 \% \mathrm{CI}$ of the dipole antenna data.

\subsection{Snow depth computation}

Again, taking advantage of the snowfall that occurred during testing, it was possible to assess snow depth with this approach; SNR data from PRN 5 from Feb. 2 to 3, between which there was a snowfall event, has been used for a comparison. The SNR data of PRN 5 before and after the snowfall are shown in Figure 16 which clearly illustrates the change in frequency expected from a height change. Figure 17 shows the antenna heights differential for the 2 days providing a relevant snow depth of $11.1 \mathrm{~cm}$. Limited access to the Table Mountain site immediately after the snow fall prevented manual verification of the snow depth. However, (1) the higher accuracy and lower variability of

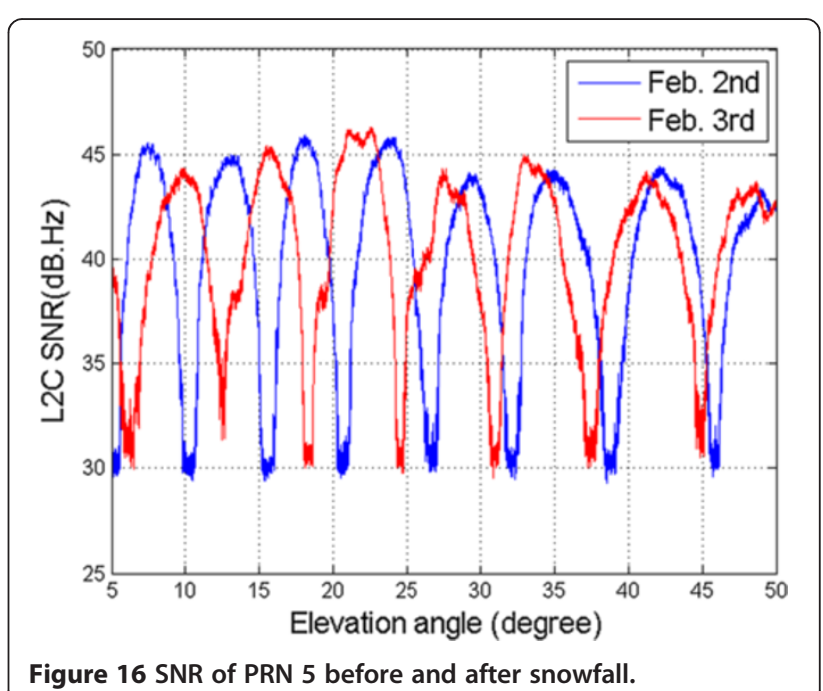




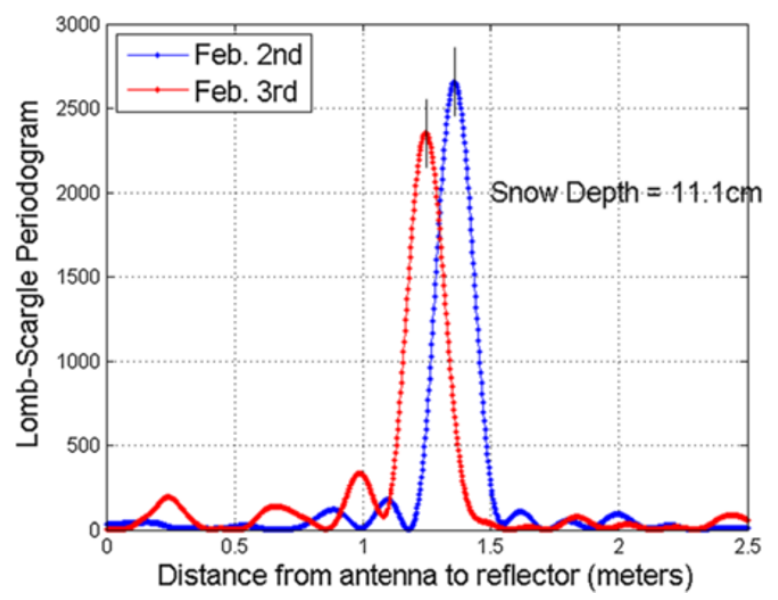

Figure 17 Lomb-Scargle periodogram before and after snowfall using PRN 5.

the bare ground measurements from the dipole when compared with the geodetic antenna and (2) the continued clear and distinct oscillation structure in the SNR for the dipole antenna in the presence of snow show the superiority of the design. To better assess and quantify the accuracy of the snow depth estimate using the dipole antenna system, a long-term experiment is underway during the 2013/2014 winter season and reference data from other snow sensors (geodetic GPS as well as laser and ultrasonic ranging) will be used.

\section{Conclusions}

In this paper, the principle of measuring snow depth via GPS-IR is reviewed and extended. In order to assess the requirements for an optimal antenna in the snow depth sensing via GPS-IR, we simulated the reflection coefficients for both horizontal and vertical polarization. Based on the analysis, the horizontally polarized electromagnetic wave is desired as a result of its constant phase of the reflection coefficient and relatively large reflection power. Given this fundamental design criteria, a dipole antenna was analyzed and built given that it is horizontally polarized around the direction at $\theta=90^{\circ}$ when horizontally oriented. Also, a L2C software-defined receiver was designed and implemented to process the raw IF signal collected by a USRP. An experiment was carried out on Table Mountain to evaluate the performance of the customized dipole antenna and SDR. The result of our dipole antenna/SDR implementation was compared with that of a geodetic GPS station with the conclusion that the dipole antenna showed significant potential for improved accuracy in snow depth sensing. Future work will focus on a long-term quantification experiment with the dipole antenna and the resulting snow depth measurements will be compared with reference data from other snow sensors.

\section{Abbreviations}

Cl: confidence interval; DLL: delay lock loop; GPS: global positioning system; GPS-IR: global positioning system - interferometric reflectometry; IF: intermediate frequency; LSP: Lomb-Scargle periodogram; LHCP: left hand circular polarized; OL: open loop; PBO: Plate Boundary Observatory; PLL: phase lock loop; RHCP: right hand circular polarized; SDR: software-defined receiver; SNR: signal-to-noise ratio; USRP: universal software radio peripheral.

\section{Competing interests}

The authors declare that they have no competing interests.

Received: 16 December 2013 Accepted: 19 June 2014

Published: 10 July 2014

\section{References}

1. J Shi, J Dozier, Estimation of snow water equivalence using SIR-C/X-SAR, Part I: inferring snow density and subsurface properties. IEEE Geosci. Rem. Sen. 38(6), 2465-2474 (2000)

2. KM Larson, ED Gutmann, VU Zavorotny, JJ Braun, MW Willimas, FG Nievinski, Can we measure snow depth with GPS receivers? Geophys. Res. Lett. 36, L17502 (2009)

3. PBO H2O Data Portal, 2013. http://xenon.colorado.edu/portal/.

4. IS-GPS-200D, Interface Specification IS-GPS-20, Revision D, Navstar GPS Space Segment/Navigation User Interfaces (Navstar GPS Joint Program Office, El Segundo, CA, 2004)

5. VU Zavorotny, KM Larson, JJ Braun, EE Small, ED Gutmann, AL Bilich, A physical model for GPS multipath caused by land reflections: toward bare soil moisture retrievals. Selected topics in applied earth observations and remote sensing. IEEE J. 3(1), 100-110 (2010)

6. KM Larson, FG Nievinski, GPS snow sensing: results from the EarthScope Plate Boundary Observatory. GPS Sol. 17(1), 41-52 (2013)

7. ED Gutmann, KM Larson, MW Williams, FG Nievinski, V Zavorotny, Snow measurement by GPS interferometric reflectometry: an evaluation at Niwot Ridge, Colorado. Hydrol. Processes 26(19), 2951-2961 (2012). doi:10.1002/hyp.8329

8. KM Larson, EE Small, E Gutman, A Bilich, P Axelrad, J Braun, Using GPS multipath to measure soil moisture fluctuations: initial results. GPS Sol. 12(3), 173-177 (2008)

9. KM Larson, EE Small, E Gutmann, A Bilich, J Braun, V Zavorotny, Use of GPS receivers as a soil moisture network for water cycle studies. Geophys. Res. Lett. 35(24), L24405 (2008)

10. Y Georgiadou, A Kleusberg, On carrier signal multipath effects in relative GPS positioning. Manuscr. Geod. 13, 172-179 (1988)

11. CJ Comp, P Axelrad, Adaptive SNR-based carrier phase multipath mitigation technique. IEEE Trans. Aerosp. Electron. Syst. 34(1), 264-276 (2008)

12. $P$ Axelrad, KM Larson, $B$ Jones, Use of the correct satellite repeat period to characterize and reduce site-specific multipath errors (ION GNSS, Long Beach, 2005)

13. A Bilich, KM Larson, Mapping the GPS multipath environment using the signal-to-noise ratio (SNR). Rad. Sci. 42, RS6003 (2007)

14. CA Balanis, Advanced Engineering Electromagnetics (John Wiley \& Sons, Inc, New York, 2005), p. 151-219. 231-275

15. P Misra, P Enge, Global Positioning System: Signals, Measurements, and Performance, 2nd edn. (Ganga-Jamuna Press, MA, 2011), pp. 398-442

16. J Norberg, GNSS Antenna Comparison for Bistatic ARK2UJ8pplication (Master's thesis) (Luleå University of Technology, 2011)

17. K Borre, D Akos, N Bertelsen, P Rinder, H Jensen Søren, Software-Defined GPS and Galileo Receiver: Single-Frequency Approach (Birkhäuser, Boston, MA, 2006)

18. KM Larson, JJ Braun, EE Small, VU Zavorotny, ED Gutman, AL Bilich, GPS multipath and its relation to near-surface soil moisture content. IEEE J. Sel. Topics Appl. Earth Obs. Remote Sens. 3(1), 91-99 (2010)

19. CZ Mooney, RD Duval, Bootstrapping: A nonparametric approach to statistical inference (Sage, Newbury Park, CA, 1993), pp. 94-95

doi:10.1186/1687-6180-2014-106

Cite this article as: Chen et al:: Snow depth sensing using the GPS L2C signal with a dipole antenna. EURASIP Journal on Advances in Signal Processing 2014 2014:106. 